\title{
Related Records Dataset
}

National Cancer Institute

\section{Source}

National Cancer Institute. Related Records Dataset. NCI Thesaurus. Code C147182.

A dataset used to describe relationships between records for a subject within or across domains, and relationships of records across datasets. (CDISC) 